\title{
Mesenchymal Stem Cells and Umbilical Cord as Sources for Schwann Cell Differentiation: their Potential in Peripheral Nerve Repair
}

\author{
Yasumasa Kuroda, Masaaki Kitada, Shohei Wakao and Mari Dezawa* \\ Department of Stem Cell Biology and Histology, Tohoku University Graduate School of Medicine, Sendai, Japan
}

\begin{abstract}
Schwann cells are important components of the peripheral glia that form myelin, serving as the microenvironment of nerve fibers in the peripheral nervous system (PNS). Damage to the PNS induces the differentiation and activation of Schwann cells to produce factors that strongly promote axonal regrowth, and subsequently contribute to remyelination, which is crucial for the recovery of function. Although the collection and transplantation of native Schwann cells are effective for the treatment of neural diseases, isolation of Schwann cells results in new damage to other peripheral nerve segments and causes undesirable iatrogenic injury in the donor. Furthermore, the expansion of native Schwann cells to obtain a sufficient number of cells for clinical application within a reasonable period is technically difficult. Therefore, a method to induce easily accessible and highly proliferative cells to differentiate into cells with Schwann cell properties would be very practical and is highly desirable. Recently, regenerative medicine has focused on mesenchymal stem cells because they are easily accessible from various kinds of mesenchymal tissues such as the umbilical cord, bone marrow, and fat tissue. Mesenchymal stem cells are highly proliferative and it is easy to obtain an adequate number of cells. Notably, while mesenchymal stem cells are mesodermal lineage cells, they have an ability to cross oligolineage boundaries previously thought uncrossable to achieve transdifferentiation. In this review, we focus on the potential of mesenchymal stem cells, particularly umbilical cord-derived mesenchymal stem cells, to differentiate into functional Schwann cells, and discuss the prospective clinical application of these cells to PNS regeneration.
\end{abstract}

Keywords: Schwann cells, differentiation, nerve regeneration, myelin, mesenchymal stem cells, umbilical cord, bone marrow, fat tissue.

\section{INTRODUCTION}

Although the peripheral nervous system (PNS) and central nervous system (CNS) are categorized into nervous tissue, nerve axons of the PNS are capable of regenerating after damage while such regeneration does not normally occur in the CNS [1]. CNS neurons, in principle, however, do have the ability to regenerate under adequate circumstances when triggered by the appropriate treatments $[2,3]$. The difference in the regenerative capacity of the PNS and CNS is explained mainly by differences in the properties of their glial cells (Fig. 1). Schwann cells, one of the most important components of the peripheral glia that forms myelin, serve as a favorable microenvironment for the repair of damaged nerve fibers in the PNS [4, 5](Fig. 1). Normally, peripheral myelin, as well as myelinating Schwann cells, inhibit axonal regeneration, but the cellular cascades that occur after damage initiate the removal of damaged myelin and the dedifferentiated Schwann cells actively produce factors that strongly promote the growth of regenerating axons $[6,7]$. On the other hand, oligodendrocytes and astrocytes are the major components of the CNS glial environment and they actively inhibit axonal regrowth. Myelin debris and reactive astrocytes are the major inhibitory factors for axonal regeneration $[8,9]$ (Fig. 1). The myelin in the CNS is constructed by oligodendrocytes and contains inhibitory

*Address correspondence to this author at the Department of Stem Cell Biology and Histology, Tohoku University Graduate School of Medicine, 21 Seiryo-machi, Aoba-ku, Sendai 980-8575, Japan; Tel: +81-22-717-8025; Fax:+81-22-717-8030; E-mail: mdezawa@med.tohoku.ac.jp molecules such as Nogo, oligodendrocyte-myelin glycoprotein, and myelin associated glycoprotein (MAG) on its surface[10]. These inhibitory molecules bind to the Nogo receptor on the surface of the distal tip of regenerating axons to transmit the inhibitory signal of axonal extension in cooperation with the co-receptors such as the low-affinity nerve growth factor receptor p75NTR, LINGO-1 (Leucinerich repeat and immunoglobulin domain-containing protein 1) and TROY (Tumor necrosis factor receptor superfamily member 19) [10]. In addition, astrocytes are activated to proliferate after injury, extend the cellular processes, and form glial scars, which physically and chemically obstruct axonal regeneration $[9,11]$.

Schwann cells that have been collected in vitro and transplanted are known to support axonal regeneration in the damaged nervous system. These cells overcome the inhibitory environment to elicit axonal regeneration and construct myelin in the CNS [12, 13]. For these reasons, Schwann cells are considered one of the most suitable cell types for inducing axonal regeneration in both the PNS and CNS.

Damage to the PNS causes axonal degeneration and demyelination, which result in functional disorder. Many approaches have been used in an attempt to restore neural function. Recent tissue engineering researches have focused on the development of bioartificial nerve conduits aimed at guiding axonal regrowth $[14,15]$. In this system, the nerve ends and intervening gap are enclosed within a tube composed of biologic or synthetic materials, thereby allowing axons to regrow into the distal nerve segment [16]. 

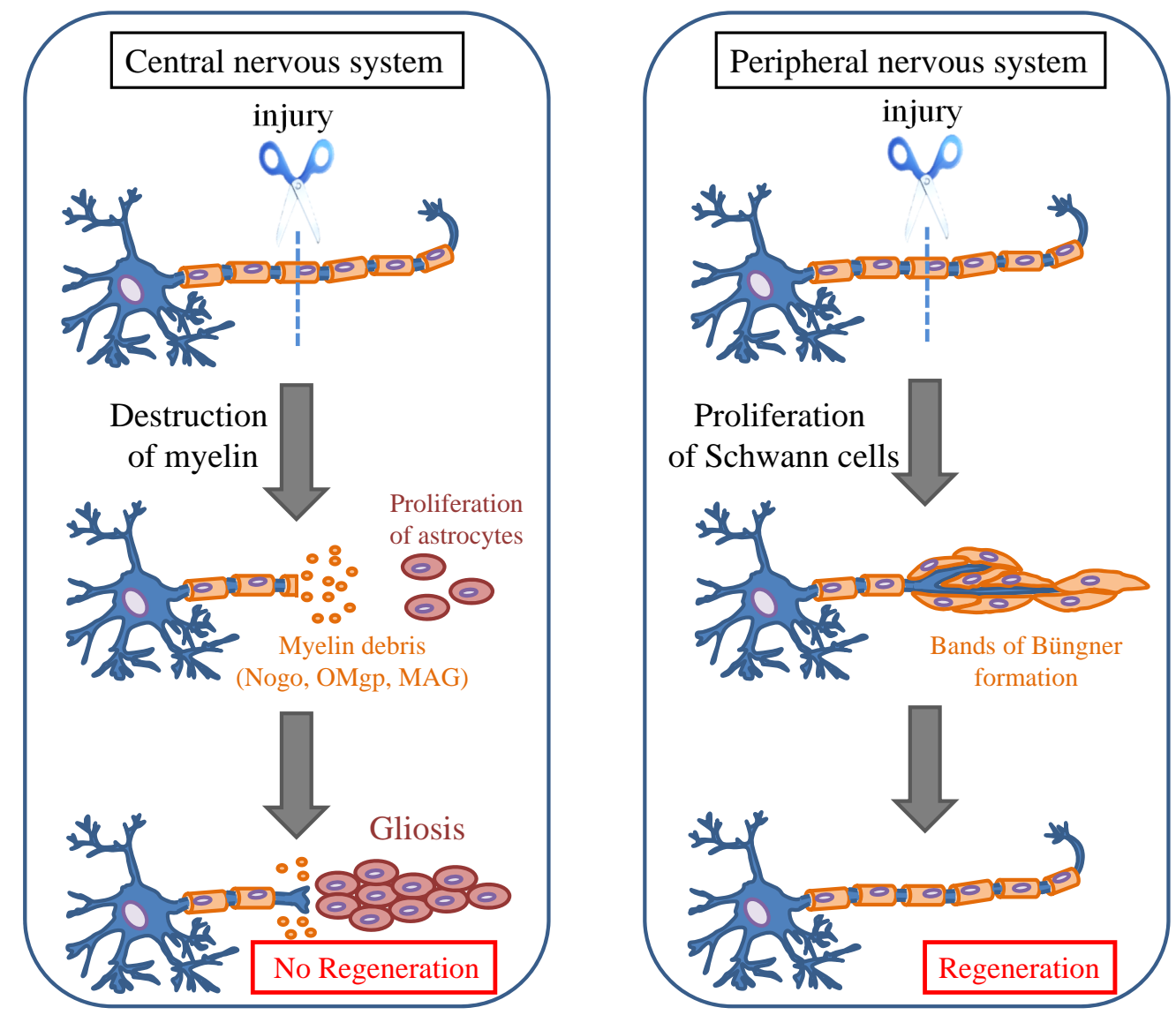

Fig. (1). Regenerative capacity of CNS and PNS. OMgp: oligodendrocyte-myelin glycoprotein, MAG: myelin associated glycoprotein.

Artificial nerve conduits are limited, however, when the nerve gap is long. In principle, Schwann cells are crucial for PNS regeneration, even when artificial nerve conduits are used; Schwann cells migrate out from both the proximal and distal nerve ends into the gap space and provide a cellular foothold to support axonal regrowth and to guide regenerating axons to the distal nerve segment where activated Schwann cells form cordons [17]. Axons successfully elongate over a long distance, mostly while in contact with cellular footholds such as Schwann cells, but long regrowth does not occur when only extracellular matrices are provided [18-20]. Therefore, when the gap is long, the limited Schwann cell migration results in limited regeneration efficiency.

Although Schwann cell collection and transplantation are effective for the treatment of neural diseases, isolation of Schwann cells causes new damage to other peripheral nerve segments, causing undesirable iatrogenic injury to the donor. Furthermore, expansion of Schwann cells to obtain an adequate cell number for clinical applications within a reasonable period of time is difficult. Therefore, a method to induce easily accessible and highly proliferative cells to differentiate into cells with Schwann cell properties would be very practical and is highly desirable.

In the past decade, researchers have focused on mesenchymal stem cells because these cells are easily accessible from various types of mesenchymal tissues and are highly proliferative so that an adequate number of cells can be obtained. Notably, while mesenchymal stem cells are meso- dermal lineage cells, they are able to cross oligolineage boundaries that were previously thought to be uncrossable to achieve transdifferentiation [21]. In bone marrow stromal cells (BMSCs), mesenchymal stem cells differentiate into endodermal cells as well as ectodermal cells, including Schwann cells [21-25]. Such multipotency has also been clarified in umbilical cord (UC)-derived mesenchymal stem cells (UC-MSCs) and adipose-derived stem cells (ADSCs), other promising mesenchymal stem cells $[26,27]$. UC-MSCs and ADSCs are also be able to differentiate into Schwann cells $[28,29]$. Here in this review, we focus on the potential of mesenchymal stem cells to differentiate into functional Schwann cells and discuss the potential clinical application of mesenchymal stem cells to PNS regeneration.

\section{REACTION OF SCHWANN CELLS TO NEURAL DAMAGE}

Damage to the PNS produces a cascade of cellular and molecular reactions in the nerve segment distal to the injury site, which is called Wallerian degeneration (originally observed by Waller, 1850) [5].

\section{Axonal Degradation}

The first step involves the prompt degradation of axons and myelin sheaths. Proximal to the site of injury, damage to nerve axons immediately activates intracellular signaling pathways and the expression of various kinds of genes related to the cell emergency system, such as c-fos and c-jun 
[30]. Free intracellular $\mathrm{Ca}^{2+}$ and calpains also act on axonal neurofilaments and microtubules, leading to axonal degradation [31, 32].

\section{Myelin Degradation}

Schwann cells that have lost axonal contact initiate the degeneration of their own myelin sheaths. This is an intrinsic process of self-destruction and is very important for subsequent regeneration [33-35]. Because myelin and myelinated Schwann cells contain axon growth inhibitors, rapid removal of myelin debris as well as changes of myelinated Schwann cells into an unmyelinated state is a key step for preconditioning the PNS toward regeneration $[6,7]$.

\section{Immune Cell Responses}

Damage triggers the infiltration of immune cells within hours. Mast cells release histamine and chemokines, which further recruit neutrophils, monocytes/macrophages, and lymphocytes [36-38]. The most important inflammatory cells during PNS regeneration are macrophages. Two kinds of macrophages are involved in regeneration; resident endoneurial macrophages, which reside within the endoneurium of PNS and are ED-2 (CD163, a cell surface glycoprotein, $175 \mathrm{kDa}$ )-positive [39, 40]; and hematogenous monocytes/ macrophages that are ED-1 (CD68, a single chain glycoprotein, 90-110kDa)-positive [41]. Resident endoneurial macrophages are activated within a couple of days after damage, and later, following the destruction of the bloodnerve barrier, hematogenous monocytes/macrophages begin to invade into the nerve segment distal to the injured site at 5 to 7 days after injury [42-44]. In addition to blood-nerve barrier destruction, the interaction between Schwann cells and resident macrophages also contributes to attracting hematogenous monocytes/macrophages [45]. For example, an autocrine cascade of interleukin-6 (IL-6) and leukemia inhibitory factor enhances Schwann cell secretion of monocyte chemoattractant protein-1, which directly attracts the infiltration of hematogenous monocytes/macrophages into the injured nerves [46-48]. In turn, macrophages secrete cytokines and trophic factors, such as IL-1 and insulin-like growth factor (IGF), which enhance the activation of Schwann cells as well as fibroblasts, followed by axonal growth [49].

Hematogenous monocytes/macrophages spread into the entire distal nerve segment by day $14[43,44]$. Both the resident endoneurial macrophages and hematogenous monocytes/macrophages are involved in the removal of myelin sheaths. Importantly, as mentioned above, not only macrophages but also Schwann cells contribute to myelin removal [33].

\section{Schwann Cell Activation}

The most characteristic feature of Wallerian degeneration is the proliferation and activation of Schwann cells within the distal nerve segment to form Schwann cell cordons, which are called bands of Büngner [5]. These Schwann cells produce various factors and cytokines that create a supportive milieu for regenerating axons in the distal segment. In the undamaged PNS, Schwann cells have a reciprocal relationship with the axons they ensheath. Axonal signals, whether acting by direct contact or by diffusible factors, regulate Schwann cell genes and control proliferation and differentiation. Conversely, Schwann cell signals regulate gene expression and intracellular axonal signaling [50]. Such a tightly regulated relationship between axons and Schwann cells is disrupted by injury, leading to subsequent Wallerian degeneration. Schwann cells quickly downregulate the expression of myelin protein genes and upregulate the low affinity neurotrophin receptor p75, as well as neurotrophins: nerve growth factor (NGF), brain-derived neurotrophic factor (BDNF), and neurotrophin-4 (NT-4) $[5,51]$. These neurotrophic factors are key regulatory proteins that modulate neuronal survival, axonal growth, synaptic plasticity, and neurotransmission. Schwann cells secrete a large variety of neurotrophic factors, including neurotrophins, transforming growth factor, basic fibroblast growth factor (bFGF), fibroblast growth factor-5, glial cell line-derived neurotrophic factor, osteopontin, IL-1 $\beta$, IL-6 and leukemia inhibitory factor. All of these factors are thought to contribute to successful axonal regeneration [5, 52-54]. Particularly, neurotrophins and their downstream Ras/mitogen activated protein kinase and phosphoinositide-3-kinase/Akt pathways are critical for both axonal growth and neuron survival in the PNS $[55,56]$.

Activated Schwann cells express a variety of cell adhesion molecules, including neural cell adhesion molecules, L1, CHL1 (close homologues of L1), N-cadherin and integrins, represented by $\alpha 1 \beta 1$ and $\alpha 6 \beta 1$-integrin, that mediate interactions between Schwann cells and axons, including growth cones $[4,17,57,58]$. In addition to these trophic factors and cell adhesion molecules, Schwann cells supply molecules of the extracellular matrix, such as fibronectin, laminin, J1/tenascin, and merosin (laminin-2), which also support the extension of regenerating axons [57]. Activated Schwann cells express connexin 46 and other connexins, coupled through junctional channels, and tight junctionrelated proteins $[18,59]$. Finally, reinnervated Schwann cells cease dividing, show downregulated expression of the molecules related to nerve regeneration, and revert to an axon-associated phenotype [5].

\section{Axonal Regeneration and Re-myelination}

Regenerating axons elongate into the distal nerve segment guided by Schwann cell cordons in the bands of Büngner. Along with axonal regeneration, reorganization of the endoneurial extracellular matrix occurs. In the later period of regeneration, Schwann cells ensheath axons and reconstruct myelin necessary for PNS function. During regeneration, one Schwann cell surrounds several regenerating axons, but eventually the cells segregate to form a $1: 1$ relationship between axon and Schwann cell, and the process of remyelination is completed [5].

\section{CELL BASED-THERAPY FOR PERIPHERAL NERVE REGENERATION USING SCHWANN CELLS DIF- FERENTIATED FROM MESENCHYMAL STEM CELLS}

In the past decade, researchers in stem cell biology and regenerative medicine have focused on mesenchymal stem cells because these cells are easily accessible from the bone 
marrow, umbilical cord, or adipose tissues, and are highly proliferative so that adequate numbers of cells can be obtained for clinical application. Furthermore, mesenchymal stem cells can be collected without encountering serious ethical problems, and there is no need to use fertilized eggs or a fetus such as in case of embryonic stem cells or neural stem/progenitor cells. Thus, mesenchymal stem cells are a strong potential candidate for use in cell-based therapy, and are already applied to patients; i.e., BMSCs for arthrosis deformans and myocardial infarction $[60,61]$. Therefore, mesenchymal stem cells are also expected to be applicable for neural regeneration.

Adult human mesenchymal stem cells originate from mesodermal lineage, but also differentiate into ectodermalas well as endodermal-lineage cells. The fact that they are able to cross oligolineage boundaries previously thought to be uncrossable stimulated development of the field of regenerative medicine and promoted studies aimed at generating desired cells from mesenchymal stem cells [21]. Particularly, BMSCs have been targeted for this research. To date, various cell types such as mesodermal lineage cells (bone, cartilage, adipocytes, skeletal muscles, and cardiomyocytes), as well as endodermal lineage cells (airway epithelial cells, hepatocytes, and insulin producing cells) and ectodermal lineage cells (neuronal cells), have been induced from BMSCs in vitro, mostly by using cytokines, trophic factors, or gene introduction [21, 23-25, 62, 63]. Recently, pluripotent stem cells, named multilineage-differentiating stress enduring (Muse) cells, were found among adult human mesenchymal stem cells such as BMSCs, dermal skin fibroblasts, and in mesenchymal tissues such as bone marrow or dermis [64]. Muse cells are capable of differentiating into cells representative of all three germ layers from a single cell, which may explain their broad spectrum of differentiation [64].

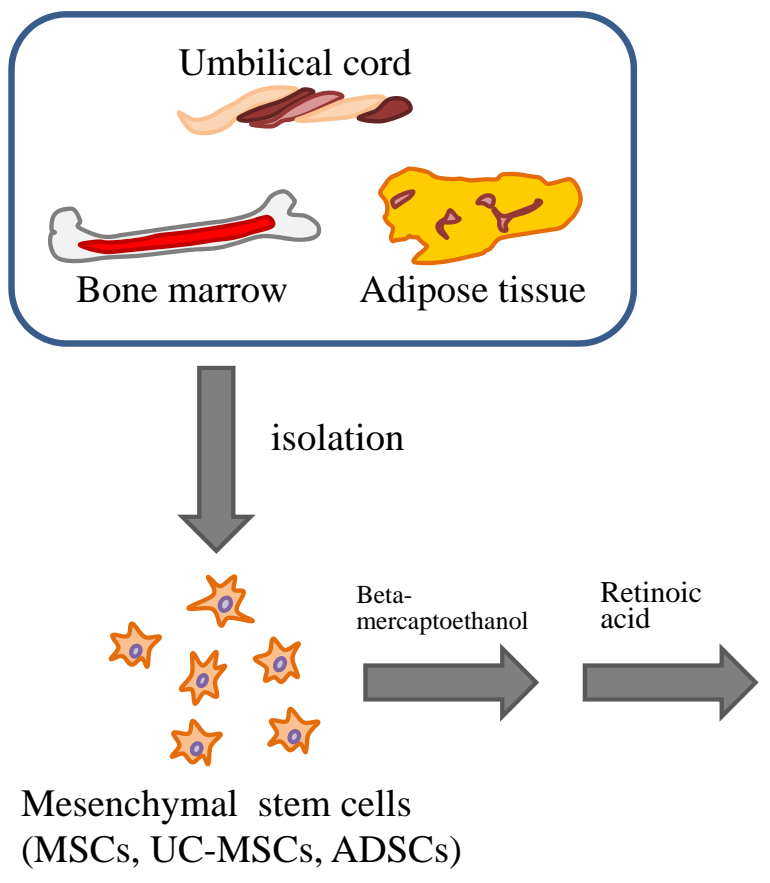

The induction system for differentiating Schwann cells from BMSCs was first reported by Dezawa et al. in 2001 [22](Fig. 2). In their report, serial administration of reagents and cytokines (described below) induced nearly $97 \%$ of BMSCs to differentiate into Schwann cell marker-positive cells, and the induced cells were shown to elicit nerve regeneration and to form myelin when transplanted into a PNS injury model. With or without some modifications, subsequent studies confirmed the successful application of this system to other kinds of mesenchymal stem cells.

Along with BMSCs, the UC and ADSCs are other promising sources of mesenchymal stem cells. Mesenchymal tissue of the UC, so-called Wharton's jelly, contains an abundance of mesenchymal stem cells. These cells have an advantage over BMSCs in that the UC derives from postnatal tissue that is discarded after birth and thus cell collection is not an invasive procedure for donors or patients. Because of the ability of UC cells to differentiate into other cell types and to proliferate, these cells are a practical and ideal source for cell-based therapies. Recently, UC-MSCs and ADSCs were shown to differentiate into Schwann cells capable of supporting neural regeneration and constructing myelin [28, 29] (Fig. 2). The following sections discuss the induction of cells exhibiting Schwann cell properties from mesenchymal stem cells, and the potential clinical application of mesenchymal stem cells to PNS regeneration.

\section{- BMSCs}

BMSCs are easily accessed by aspiration of the bone marrow. They can be isolated from patients and expanded in a large scale, both from healthy donors and patients. For example, 20 to $100 \mathrm{ml}$ of bone marrow aspirate yields $1 \times 10^{7}$ of BMSCs within several weeks, which provides a sufficient number of cells for transplantation.

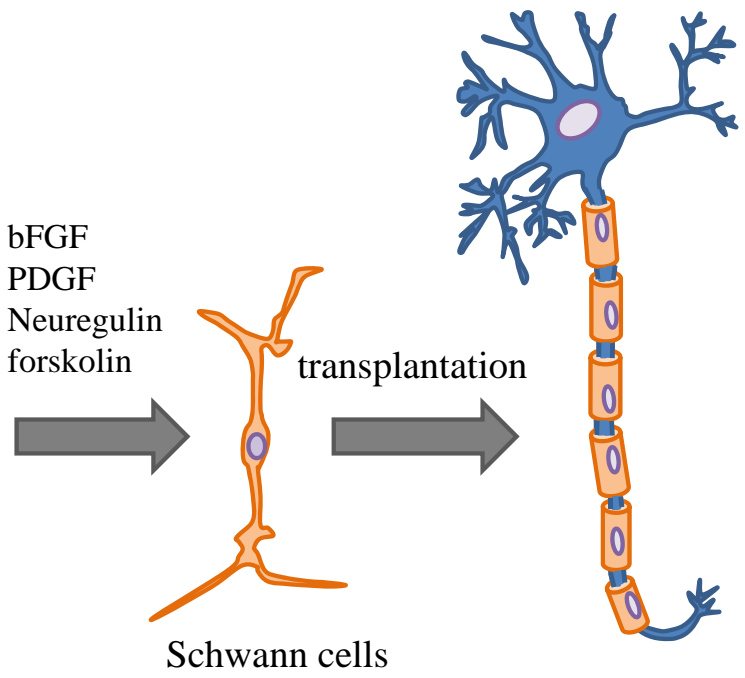

Fig. (2). Schwann cells can be differentiated from mesenchymal stem cells of bone marrow, umbilical cord and adipose tissue. 
Dezawa et al., (2001) were the first to attempt the induction of Schwann cells from mesenchymal stem cells using BMSCs [22]. The sequential treatment of rat BMSCs dispersed at a certain cell density with the reducing reagent beta-mercaptoethanol, all-trans-retinoic acid, and then a mixture of forskolin (known to upregulate intracellular cAMP concentration), bFGF, platelet-derived growth factor (PDGF), and neuregulin-1 (NRG-1 [isoforms include Type I NRG1, also called heregulin, and Type II NRG1, also called glial growth factor-2 (GGF-2)]) generated cells with Schwann cell properties with very high efficiency ( $\sim 97 \%)$. The differentiated cells exhibited Schwann cell properties and expressed Schwann cell markers such as protein zero (P0), low-affinity nerve growth factor receptor $\mathrm{p} 75$, glial fibrillary acidic protein, S-100, and $\mathrm{O} 4$ [22]. Importantly, differentiated Schwann cells contributed to axonal regeneration as well as to reconstruction of myelin when transplanted into transected rat peripheral nerve, which is the most important property of native Schwann cells in PNS injury $[22,65]$.

This induction method was further reproduced by several groups, and is now widely applied to Schwann cell induction from a wide range of mesenchymal stem cells, including BMSCs, UC-MSCs and ADSCs [28, 66-69]. Schwann cells differentiated from BMSCs support axonal regeneration both in vitro and in vivo. In co-culture with dorsal root ganglion neuron, differentiated Schwann cells substantially support neurite outgrowth[70-72]. When differentiated Schwann cells are transferred into conduits and transplanted into the transected gap made in peripheral nerve, they sustain axonal regrowth and formed myelin, leading to functional recovery in nerve conduction velocity, walking track analysis, average myelin area, and myelinated axon count $[22,65,73]$. Chitosan is shown to be a favorable conduit to fill with differentiated Schwann cells for transplantation [74]. Histologically, transplanted cells were confirmed to form myelin and express myelin-related proteins such as MAG, myelinbasic protein, P0 or peripheral myelin protein 22 (PMP22) $[22,65,75,76]$.

The effectiveness of Schwann cells differentiated from BMSCs was further demonstrated in human BMSCs; under the control of immunosuppressants, these cells support axonal regeneration, construct the myelin sheath, and lead to the recovery of sciatic nerve function when transplanted into a rat sciatic nerve transection model [77]. Preclinical studies using cynomolgus monkeys demonstrated that, in behavior analysis, electrophysiology, and histologic evaluation, autologous transplantation of Schwann cells differentiated from BMSCs is effective for accelerating the regeneration of transected axons and for functional recovery of injured nerves. Furthermore, ${ }^{18}$ F-fluorodeoxyglucose-positron emission tomography scanning demonstrated no abnormal accumulation of radioactivity except in regions with an expected physiologic accumulation up to 1 year and thus confirmed the safety of this transplantation treatment [78].

These findings described above indicate that the practical advantages of BMSCs are expected to make this system applicable for neurotrauma or peripheral nerve disorders where the acceleration of regeneration is expected to enhance functional recovery.

\section{- UC-MSCs}

The UC-MSCs are highly advantageous as potential sources for cell-based therapies because of their easy accessibility and the few ethical problems regarding their use, as long as donors provide informed consent. UC-MSCs are proliferative cells, and cell collection is not an invasive procedure for donors or patients. They are available from cord bank. Tumorigenesis in transplantation experiments of UC-MSCs and of UC-MSC-differentiated cells is rare[79, 80], and these cells have the potential to differentiate into a wide variety of cell types as reported previously[81-84]. For example, neural cells including neurons, oligodendrocytes and astrocytes [85] and even endodermal cell types such as hepatocytes and pancreatic beta cells can be given rise to from UC-MSCs [86, 87]. Thus, along with BMSCs, UCMSCs are considered one of the most practical sources for cell-based therapies.

Several groups have demonstrated the differentiation of functional Schwann cells from human UC-MSCs. Dezawa's group showed that the method used for BMSCs, i.e., betamercaptoethanol, retinoic acid and the mixture of bFGF, PDGF, and the heregulin-A1-EGF domain together with forskolin successfully induced the differentiation of Schwann cells from human UC-MSCs (UC-Schwann cells) with very high efficiency ( $\sim 97 \%)$, and that UC-Schwann cell function is comparable to that of native human Schwann cells in vivo[29]. Transplantation into rat transected sciatic nerve under the control of immunosuppressants showed that the human UC-Schwann cells maintained their differentiated phenotype in vivo after transplantation and contributed to axonal regeneration and functional recovery. The transplanted UC-Schwann cells, which were pre-labeled with lentivirus green fluorescent protein, expressed MAG, PMP22, and periaxin (a marker of peripheral myelin), and immunoelectron microscopy revealed that transplanted cells formed the myelin of regenerated axons. Consistent with these results, functional recovery (measured by walking track analysis) achieved by the transplantation of UCSchwann cells was almost identical to that of native human Schwann cells [29]. Other groups demonstrated that UCSchwann cells differentiated by the same method produced neurotrophic factors such as NGF and BDNF [66, 67, 88]. These findings indicated that UC-Schwann cells are a viable alternative to native Schwann cells and may be applied to cell-based therapy for nerve injuries and degenerative diseases.

\section{- ADSCs}

Mesenchymal stem cells prepared from adipose tissue are referred to as ADSCs. The properties of ADSCs are similar to BMSCs in that they also have the capacity to differentiate into various kinds of cells [89-92]. Because humans have abundant sources for ADSCs (i.e., subcutaneous fat deposits that can be isolated by conventional liposuction procedures), they are also expected to be a practical cell source for cellbased therapy.

Terenghi's group was the first to report Schwann cell differentiation from ADSCs in 2007 [28]. They obtained Schwann cells by treating ADSCs with beta-mercaptoethanol and retinoic acid, followed by a mixture of bFGF, PDGF, 
forskolin, and neuregulin (GGF-2). The differentiated cells expressed Schwann cell markers and coculture with neuronal cells induced neurite outgrowth. Haycock et al., (2011) reproduced these data, and further clarified that perinephric ADSCs have high potential to become Schwann cells compared with ADSCs from other sources such as subcutaneous or epididymal fat tissues [69].

Other differentiation systems have been developed as well; Vogt et al., (2009) demonstrated that culturing ADSCs in neurosphere culture followed by dissociation of the formed spheres and the removal of mitogen results in the differentiation of ADSCs into Schwann cell-like cells that are positive for $\mathrm{p} 75, \mathrm{~S}-100$, and glial fibrillary acidic protein [93]. Zhang et al., (2008) reported that co-culturing ADSCs with native Schwann cells is effective for Schwann cell differentiation [94].

Because the harvest of BMSCs is an invasive and painful procedure, and UC-MSCs are usually not applicable to autologous transplantation, ADSCs will be useful alternative cells for Schwann cell production and cell-based therapy.

\section{THE MECHANISMS OF SCHWANN CELL DIFFER- ENTIATION; STUDIES OF UC-SCHWANN CELLS}

The method used to induce Schwann cells, namely, treatment of cells first with beta-mercaptoethanol, then with retinoic acid, and finally with a mixture of bFGF, forskolin, PDGF, and neuregulin seems to apply generally to mesenchymal stem cells. The rational for this induction system was considered in UC-Schwann cell differentiation [29].

Exposure of UC-MSCs to beta-mercaptoethanol and retinoic acid is a prerequisite because elimination of these factors fails to differentiate into Schwann cells. The betamercaptoethanol acts as a reducing agent on BMSCs to promote differentiation into neural-lineage cells by the synthesis of glutathione [95, 96]. Retinoic acid is a wellknown factor that acts as a morphogen during development, regulating the expression of various transcription factors that are crucial for early neural determination such as MASH1 and NeuroD, and has a role in the acquisition of the responsiveness to neurotrophins [97-99].

The bFGF functions as a mitogen and accelerates Schwann cell precursors during Schwann cell transition $[100,101]$. PDGF contributes to DNA synthesis and acts as a mitogen in Schwann cells [102]. Neuregulin, either heregulin or GGF, selectively induces Schwann cells from neural crest cells and promotes the survival and proliferation of Schwann cell progenitors [100]. Forskolin increases the level of intracellular cyclic adenosine monophosphate and the expression level of growth factor receptors. Therefore, the addition of forskolin to the combination of bFGF, PDGF, and heregulin might enhance cellular responses to trophic factors, leading to efficient trophic factor stimulation for Schwann cell differentiation [103].

Interestingly, gene expression studies demonstrated that UC-MSCs were initially negative for P0 and S-100B and slightly positive for Sox 10 and Krox20 [29]. After the induction of UC-Schwann cells, P0 and S-100B began to be expressed, and expression of Sox10 and Krox20 was substantially upregulated. In contrast, the expression of a cell marker for the immature neural lineage Hath1 was initially positive in UC-MSCs, but decreased after the induction process, suggesting that UC-Schwann cells undergo sequential differentiation through this induction process [29].

In summary, beta-mercaptoethanol and retinoic acid might work together to trigger factors that alter the characteristics of UC-MSCs to those of neural lineage cells, and subsequent treatment with forskolin, bFGF, PDGF, and neuregulin synergistically promote the differentiation of UCMSCs into cells with Schwann cell characteristics. Therefore, the use of these factors for inducing cells with Schwann cell properties mimics normal Schwann cell development and thus this system is generally efficient for differentiation of mesenchymal stem cells into Schwann cells.

\section{PERSPECTIVES}

Vigorous axonal regeneration can be elicited when the injured PNS is provided a cellular foothold with the ability to support axonal growth. Compared to biomaterial nerve conduits, the number of regenerating axons is more abundant, and the regeneration speed is faster when the damaged PNS is provided with this cellular foothold. Furthermore, simple axonal regrowth is not sufficient for PNS regeneration. Reconstruction of myelin is necessary for the procurement of saltatory conduction, which is critical for functional recovery. For these reasons, Schwann cells are the most crucial cells for cell-based therapy in PNS regeneration. Transplantation of Schwann cells in combination with the use of biomaterials that are effective for supporting neural regeneration strongly promotes regeneration efficiency.

Schwann cells originate from neural crest cells [54]. Although cells with neural crest origin such as skin-derived precursors are reported to reside in adult tissues[104], these cells are technically difficult to obtain from adult tissues in large numbers for clinical application. Alternative sources are native Schwann cells that can be harvested from the adult human PNS system. It is difficult, however, to obtain an adequate amount of Schwann cells from the human PNS for clinical use. Besides, Schwann cells cannot be harvested without damaging healthy peripheral nerve. Thus, it would be more desirable to establish cells with Schwann cell characteristics from sources that are easily accessible and capable of rapid expansion.

Mesenchymal stem cells offer great potential for cell transplantation therapy because of their easy accessibility and proliferative capacity. Undifferentiated naive BMSCs or UC-MSCs have limited efficiency for PNS regeneration; compared to the transplantation of Schwann cells differentiated from BMSCs or UC-MSCs, the number of regenerating axons and the extent of functional recovery are far below that of transplantation with naive BMSCs or UCMSCs [29, 65, 77, 78]. Therefore, it is rational to use differentiated Schwann cells, but their practical application to human PNS disorders is dependent on the technique used to control their differentiation into functional Schwann cells with high efficiency and purity. In many cases, differentiation of MSCs involves the introduction of specific genes. The basic method of differentiating Schwann cells from 
MSCs (beta-mercapthoethanol -> retinoic acid -> bFGF+ $\mathrm{PDGF}+$ forskolin+neuregulin) [22], however, involves only the reagent and trophic factor administration and not any gene introduction.

The function of Schwann cells in neural regeneration is primarily a facilitatory effect for axonal regrowth as well as for the reconstruction of myelin to allow for saltatory conduction in the PNS. Therefore, differentiated Schwann cells must not only express markers for Schwann cells and myelin, but must also have the ability to form myelin, and this characteristic is critical for the evaluation of differenttiated cells. In this sense, Schwann cells differentiated from BMSCs, UC-MSCs, and ADSCs compare favorably with native Schwann cells. Notably, differentiated Schwann cells obtained from monkey BMSCs were demonstrated to be effective and safe for PNS regeneration up to 1 year when evaluated based on general condition, tumor markers in blood analysis, and 18F-fluorodeoxyglucose-positron emission tomography scanning [78]. Furthermore, for clinical application, it is important that not only rodent cells but also human BMSCs, UC-MSCs, and ADSCs are capable of differentiating into cells with Schwann cell properties.

For these reasons, Schwann cells differentiated from human mesenchymal stem cells seem to have the most appropriate properties for clinical application.

Auto cell transplantation is an ideal therapy for regenerative medicine because it has carries less risk of rejection and infection. BMSCs and ADSCs are practical cells that are expected to be applicable for auto cell transplantation therapy in neurodegenerative diseases [105] (Fig. 3). Some patients, however, will not be able to use their own cells due to older age or underlying disease. In such circumstances, UC-MSCs might be an alternative choice for cell-based therapy (Fig. 3). Furthermore, UC-MSCs are superior to other cell sources in some respects because they can be noninvasively collected from many volunteers and cord banks that have been already established. A potential disadvantage of using UC-MSCs, as in allogenic transplantation, is the need for continuous immunosuppression in case of an HLA mismatch. Mesenchymal stem cells, however, suppress immunoresponses, known as immunomodulation, and in fact, allogenic BMSCs are already used in clinical application for treating graft-versus-host disease[106]. Although more studies are currently needed to demonstrate that UC-Schwann cells inherit the character of MSCs in terms of immunomodulation, UC-Schwann cells might be applicable for allogenic transplantation.

Previous studies indicate that Schwann cells also support axonal regeneration, construct myelin, and contribute to functional recovery in a spinal cord injury model [107-109]. The results suggest that Schwann cells differentiated from mesenchymal stem cells such as BMSCs, UC-MSCs, and ADSCs may also be effective for therapeutic application to spinal cord injury. In addition, other animal models of peripheral nerve injury resembling clinical cases, such as crush or laceration, may be more informative for the assessment of mesenchymal stem cells-derived Schwann cell therapies.

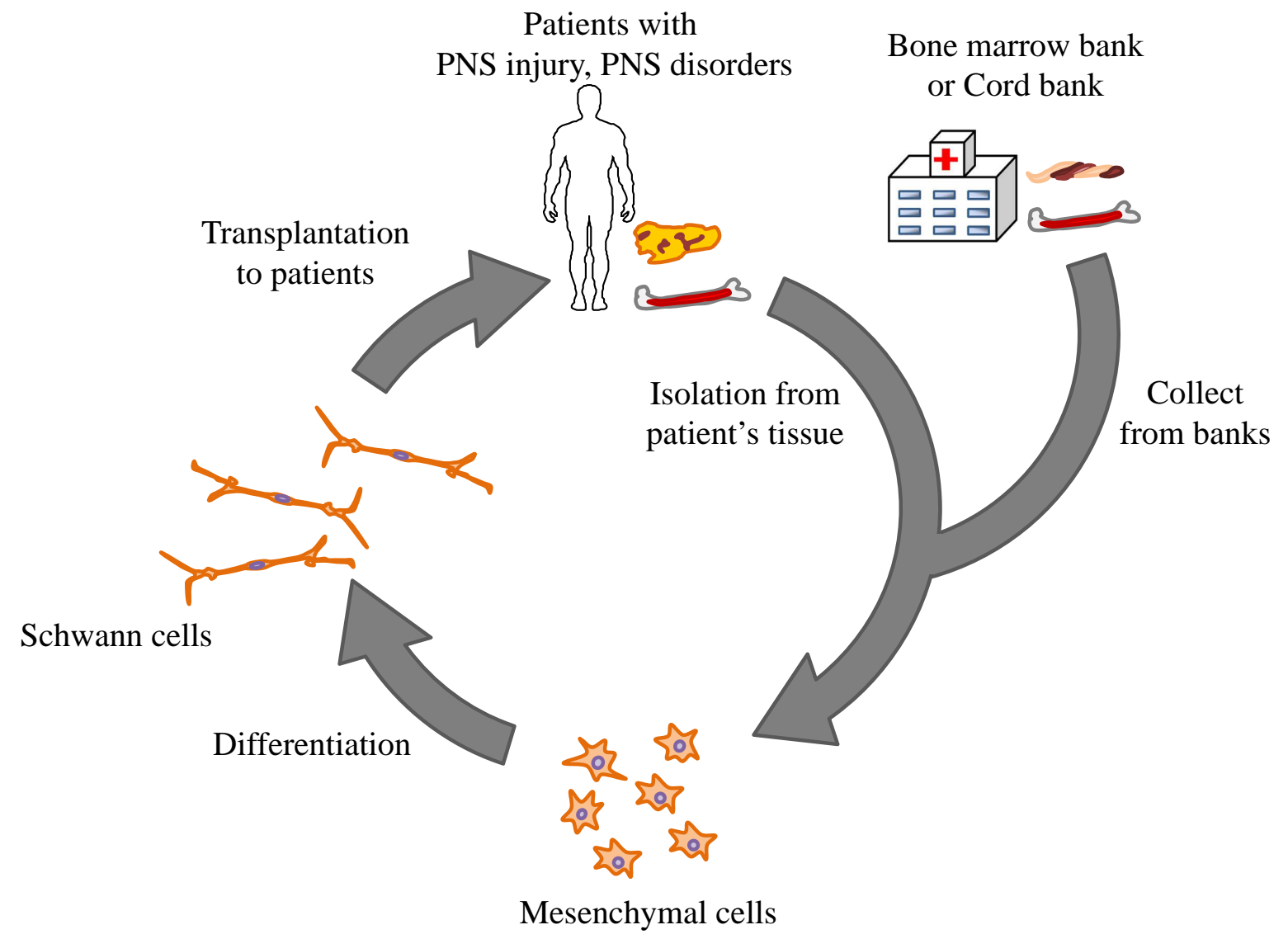

Fig. (3). Strategy for clinical application of Schwann cells differentiated from mesenchymal stem cells. 


\section{ACKNOWLEDGEMENTS}

This study was supported by Program for the Promotion of Fundamental Studies in Health Sciences of the National Institute of Biomedical Innovation (NIBIO).

\section{REFERENCES}

[1] David S, Aguayo AJ. Axonal regeneration after crush injury of rat central nervous system fibres innervating peripheral nerve grafts. J Neurocytol 1985; 14(1): 1-12.

[2] So KF, Aguayo AJ. Lengthy regrowth of cut axons from ganglion cells after peripheral nerve transplantation into the retina of adult rats. Brain Res 1985; 328(2): 349-54

[3] Dezawa M, Adachi-Usami E. Role of Schwann cells in retinal ganglion cell axon regeneration. Prog Retin Eye Res 2000; 19(2): 171-204.

[4] Martini R. Expression and functional roles of neural cell surface molecules and extracellular matrix components during development and regeneration of peripheral nerves. J Neurocytol 1994; 23(1): 1-28.

[5] Hall S. Nerve repair: a neurobiologist's view. J Hand Surg Br 2001; 26(2): 129-36.

[6] Barrette B, Hebert MA, Filali M, et al. Requirement of myeloid cells for axon regeneration. J Neurosci 2008; 28(38): 9363-76.

[7] Shen YJ, DeBellard ME, Salzer JL, Roder J, Filbin MT. Myelinassociated glycoprotein in myelin and expressed by Schwann cells inhibits axonal regeneration and branching. Mol Cell Neurosci 1998; 12(1-2): 79-91.

[8] Llorens F, Gil V, del Rio JA. Emerging functions of myelinassociated proteins during development, neuronal plasticity, and neurodegeneration. FASEB J 2011; 25(2): 463-75.

[9] Buffo A, Rite I, Tripathi P, et al. Origin and progeny of reactive gliosis: A source of multipotent cells in the injured brain. Proc Natl Acad Sci USA 2008; 105(9): 3581-6.

[10] Zorner B, Schwab ME. Anti-Nogo on the go: from animal models to a clinical trial. Ann N Y Acad Sci 2010; 1198 (Suppl 1): E22-34.

[11] Pekny M, Pekna M. Astrocyte intermediate filaments in CNS pathologies and regeneration. J Pathol 2004; 204(4): 428-37.

[12] Bunge MB. Transplantation of purified populations of Schwann cells into lesioned adult rat spinal cord. J Neurol 1994; 242(1 Suppl 1): S36-9.

[13] Harvey AR, Plant GW, Tan MM. Schwann cells and the regrowth of axons in the mammalian CNS: a review of transplantation studies in the rat visual system. Clin Exp Pharmacol Physiol 1995; 22(8): 569-79.

[14] Ishikawa N, Suzuki Y, Ohta M, et al. Peripheral nerve regeneration through the space formed by a chitosan gel sponge. J Biomed Mater Res A 2007; 83(1): 33-40.

[15] Ohta M, Suzuki Y, Chou H, et al. Novel heparin/alginate gel combined with basic fibroblast growth factor promotes nerve regeneration in rat sciatic nerve. J Biomed Mater Res A 2004; 71(4): 661-8.

[16] Gu X, Ding F, Yang Y, Liu J. Construction of tissue engineered nerve grafts and their application in peripheral nerve regeneration. Prog Neurobiol 2011; 93(2): 204-30.

[17] Ide C. Peripheral nerve regeneration. Neurosci Res 1996; 25(2): 101-21.

[18] Dezawa M, Nagano T. Contacts between regenerating axons and the Schwann cells of sciatic nerve segments grafted to the optic nerve of adult rats. J Neurocytol 1993; 22(12): 1103-12.

[19] Dezawa M, Kawana K, Adachi-Usami E. The role of Schwann cells during retinal ganglion cell regeneration induced by peripheral nerve transplantation. Invest Ophthalmol Vis Sci 1997; 38(7): 1401-10.

[20] Dezawa M, Kawana K, Negishi H, Adachi-Usami E. Glial cells in degenerating and regenerating optic nerve of the adult rat. Brain Res Bull 1999; 48(6): 573-9.

[21] Pittenger MF, Mackay AM, Beck SC, et al. Multilineage potential of adult human mesenchymal stem cells. Science 1999; 284(5411): 143-7.

[22] Dezawa M, Takahashi I, Esaki M, Takano M, Sawada H. Sciatic nerve regeneration in rats induced by transplantation of in vitro differentiated bone-marrow stromal cells. Eur J Neurosci 2001; 14(11): 1771-6.
[23] Dezawa M, Kanno H, Hoshino M, et al. Specific induction of neuronal cells from bone marrow stromal cells and application for autologous transplantation. J Clin Invest 2004; 113(12): 1701-10.

[24] Dezawa M, Ishikawa H, Itokazu Y, et al. Bone marrow stromal cells generate muscle cells and repair muscle degeneration. Science 2005; 309(5732): 314-7.

[25] Oyagi S, Hirose M, Kojima M, et al. Therapeutic effect of transplanting HGF-treated bone marrow mesenchymal cells into CCl4-injured rats. J Hepatol 2006; 44(4): 742-8.

[26] Wang Y, Zhao L, Hantash BM. Support of human adipose-derived mesenchymal stem cell multipotency by a poloxamer-octapeptide hybrid hydrogel. Biomaterials 2010; 31(19): 5122-30.

[27] Troyer DL, Weiss ML. Wharton's jelly-derived cells are a primitive stromal cell population. Stem Cells 2008; 26(3): 591-9.

[28] Kingham PJ, Kalbermatten DF, Mahay D, Armstrong SJ, Wiberg $\mathrm{M}$, Terenghi G. Adipose-derived stem cells differentiate into a Schwann cell phenotype and promote neurite outgrowth in vitro. Exp Neurol 2007; 207(2): 267-74.

[29] Matsuse D, Kitada M, Kohama M, et al. Human umbilical cordderived mesenchymal stromal cells differentiate into functional Schwann cells that sustain peripheral nerve regeneration. J Neuropathol Exp Neurol 2010; 69(9): 973-85.

[30] Soares HD, Chen SC, Morgan JI. Differential and prolonged expression of Fos-lacZ and Jun-lacZ in neurons, glia, and muscle following sciatic nerve damage. Exp Neurol 2001; 167(1): 1-14.

[31] Glass JD, Culver DG, Levey AI, Nash NR. Very early activation of $\mathrm{m}$-calpain in peripheral nerve during Wallerian degeneration. J Neurol Sci. 2002; 196(1-2): 9-20.

[32] Coleman M. Axon degeneration mechanisms: commonality amid diversity. Nat Rev Neurosci 2005; 6(11): 889-98.

[33] Stoll G, Griffin JW, Li CY, Trapp BD. Wallerian degeneration in the peripheral nervous system: participation of both Schwann cells and macrophages in myelin degradation. J Neurocytol 1989; 18(5): 671-83.

[34] Bruck W. The role of macrophages in Wallerian degeneration. Brain Pathol 1997; 7(2): 741-52.

[35] Stoll G, Muller HW. Nerve injury, axonal degeneration and neural regeneration: basic insights. Brain Pathol 1999; 9(2): 313-25.

[36] Monk KR, Wu J, Williams JP, et al. Mast cells can contribute to axon-glial dissociation and fibrosis in peripheral nerve. Neuron Glia Biol 2007; 3(3): 233-44.

[37] Zuo Y, Perkins NM, Tracey DJ, Geczy CL. Inflammation and hyperalgesia induced by nerve injury in the rat: a key role of mast cells. Pain 2003; 105(3): 467-79.

[38] Moalem G, Xu K, Yu L. T lymphocytes play a role in neuropathic pain following peripheral nerve injury in rats. Neuroscience 2004; 129(3): 767-77.

[39] Griffin JW, George R, Ho T. Macrophage systems in peripheral nerves. A review. J Neuropathol Exp Neurol 1993; 52(6): 553-60.

[40] Monaco S, Gehrmann J, Raivich G, Kreutzberg GW. MHCpositive, ramified macrophages in the normal and injured rat peripheral nervous system. J Neurocytol. 1992; 21(9): 623-34.

[41] Dijkstra CD, Damoiseaux JG. Macrophage heterogeneity established by immunocytochemistry. Prog Histochem Cytochem 1993; 27(2): 1-65.

[42] Taskinen HS, Roytta M. The dynamics of macrophage recruitment after nerve transection. Acta Neuropathol 1997; 93(3): 252-9.

[43] Mueller M, Leonhard C, Wacker K, et al. Macrophage response to peripheral nerve injury: the quantitative contribution of resident and hematogenous macrophages. Lab Invest 2003; 83(2): 175-85.

[44] Omura T, Omura K, Sano M, Sawada T, Hasegawa T, Nagano A. Spatiotemporal quantification of recruit and resident macrophages after crush nerve injury utilizing immunohistochemistry. Brain Res 2005; 1057(1-2): 29-36.

[45] Gillen C, Jander S, Stoll G. Sequential expression of mRNA for proinflammatory cytokines and interleukin-10 in the rat peripheral nervous system: comparison between immune-mediated demyelination and Wallerian degeneration. J Neurosci Res 1998; 51(4): 489-96.

[46] Tofaris GK, Patterson PH, Jessen KR, Mirsky R. Denervated Schwann cells attract macrophages by secretion of leukemia inhibitory factor (LIF) and monocyte chemoattractant protein-1 in a process regulated by interleukin-6 and LIF. J Neurosci 2002; 22(15): 6696-703. 
[47] Taskinen HS, Roytta M. Increased expression of chemokines (MCP-1, MIP-1alpha, RANTES) after peripheral nerve transection. J Peripher Nerv Syst. 2000; 5(2): 75-81.

[48] Toews AD, Barrett C, Morell P. Monocyte chemoattractant protein 1 is responsible for macrophage recruitment following injury to sciatic nerve. J Neurosci Res 1998; 53(2): 260-7.

[49] Lindholm D, Heumann R, Meyer M, Thoenen H. Interleukin-1 regulates synthesis of nerve growth factor in non-neuronal cells of rat sciatic nerve. Nature 1987; 330(6149): 658-9.

[50] Bolin LM, Shooter EM. Neurons regulate Schwann cell genes by diffusible molecules. J Cell Biol 1993; 123(1): 237-43.

[51] Martinez AM. Distribution of sodium and potassium channels as well as myelin associated glycoprotein (MAG) during the early stages of Wallerian degeneration. J Submicrosc Cytol Pathol 1999; 31(1): 73-81.

[52] Abe K, Namikawa K, Honma M, et al. Inhibition of Ras extracellular-signal-regulated kinase (ERK) mediated signaling promotes ciliary neurotrophic factor (CNTF) expression in Schwann cells. J Neurochem 2001; 77(2): 700-3.

[53] Jander S, Bussini S, Neuen-Jacob E, et al. Osteopontin: a novel axon-regulated Schwann cell gene. J Neurosci Res 2002; 67(2): 156-66.

[54] Dubovy P. Wallerian degeneration and peripheral nerve conditions for both axonal regeneration and neuropathic pain induction. Ann Anat 2011; 193(4): 267-75.

[55] Goldberg JL, Barres BA. The relationship between neuronal survival and regeneration. Annu Rev Neurosci 2000; 23: 579-612.

[56] Lonze BE, Riccio A, Cohen S, Ginty DD. Apoptosis, axonal growth defects, and degeneration of peripheral neurons in mice lacking CREB. Neuron 2002; 34(3): 371-85.

[57] Chernousov MA, Carey DJ. Schwann cell extracellular matrix molecules and their receptors. Histol Histopathol 2000; 15(2): 593601.

[58] Zhang Y, Roslan R, Lang D, Schachner M, Lieberman AR, Anderson PN. Expression of CHL1 and L1 by neurons and glia following sciatic nerve and dorsal root injury. Mol Cell Neurosci 2000; 16(1): 71-86.

[59] Dezawa M, Mutoh T, Dezawa A, Ishide T. Tight junctions between the axon and Schwann cell during PNS regeneration. Neuroreport 1996; 7(11): 1829-32

[60] Kawate $\mathrm{K}$, Yajima $\mathrm{H}$, Ohgushi $\mathrm{H}$, et al. Tissue-engineered approach for the treatment of steroid-induced osteonecrosis of the femoral head: transplantation of autologous mesenchymal stem cells cultured with beta-tricalcium phosphate ceramics and free vascularized fibula. Artif Organs 2006; 30(12): 960-2.

[61] Schachinger V, Erbs S, Elsasser A, et al. Intracoronary bone marrow-derived progenitor cells in acute myocardial infarction. $\mathrm{N}$ Engl J Med 2006; 355(12): 1210-21.

[62] Makino S, Fukuda K, Miyoshi S, et al. Cardiomyocytes can be generated from marrow stromal cells in vitro. J Clin Invest 1999; 103(5): 697-705.

[63] Spees JL, Olson SD, Ylostalo J, et al. Differentiation, cell fusion, and nuclear fusion during ex vivo repair of epithelium by human adult stem cells from bone marrow stroma. Proc Natl Acad Sci USA 2003; 100(5): 2397-402.

[64] Kuroda Y, Kitada M, Wakao S, et al. Unique multipotent cells in adult human mesenchymal cell populations. Proc Natl Acad Sci USA 2010; 107(19): 8639-43.

[65] Mimura T, Dezawa M, Kanno H, Sawada H, Yamamoto I. Peripheral nerve regeneration by transplantation of bone marrow stromal cell-derived Schwann cells in adult rats. J Neurosurg 2004; 101(5): 806-12.

[66] Peng J, Wang Y, Zhang L, et al. Human umbilical cord Wharton's jelly-derived mesenchymal stem cells differentiate into a Schwanncell phenotype and promote neurite outgrowth in vitro. Brain Res Bull 2011; 84(3): 235-43.

[67] Jiang TM, Yang ZJ, Kong CZ, Zhang HT. Schwann-like cells can be induction from human nestin-positive amniotic fluid mesenchymal stem cells. In vitro Cell Dev Biol Anim 2010; 46(9): 793-800.

[68] Jiang L, Zhu JK, Liu XL, Xiang P, Hu J, Yu WH. Differentiation of rat adipose tissue-derived stem cells into Schwann-like cells in vitro. Neuroreport 2008; 19(10): 1015-9.

[69] Kaewkhaw R, Scutt AM, Haycock JW. Anatomical site influences the differentiation of adipose-derived stem cells for Schwann-cell phenotype and function. Glia 2011; 59(5): 734-49.
[70] Brohlin M, Mahay D, Novikov LN, et al. Characterisation of human mesenchymal stem cells following differentiation into Schwann cell-like cells. Neurosci Res 2009; 64(1): 41-9.

[71] Mahay D, Terenghi G, Shawcross SG. Schwann cell mediated trophic effects by differentiated mesenchymal stem cells. Exp Cell Res 2008; 314(14): 2692-701.

[72] Faroni A, Mantovani C, Shawcross SG, Motta M, Terenghi G, Magnaghi V. Schwann-like adult stem cells derived from bone marrow and adipose tissue express gamma-aminobutyric acid type B receptors. J Neurosci Res 2011.

[73] Wang X, Luo E, Li Y, Hu J. Schwann-like mesenchymal stem cells within vein graft facilitate facial nerve regeneration and remyelination. Brain Res 2011; 1383: 71-80

[74] Ao Q, Fung CK, Tsui AY, et al. The regeneration of transected sciatic nerves of adult rats using chitosan nerve conduits seeded with bone marrow stromal cell-derived Schwann cells. Biomaterials 2011; 32(3): 787-96.

[75] Krampera M, Marconi S, Pasini A, et al. Induction of neural-like differentiation in human mesenchymal stem cells derived from bone marrow, fat, spleen and thymus. Bone 2007; 40(2): 382-90.

[76] Lin W, Chen X, Wang X, Liu J, Gu X. Adult rat bone marrow stromal cells differentiate into Schwann cell-like cells in vitro. In vitro Cell Dev Biol Anim 2008; 44(1-2): 31-40.

[77] Shimizu S, Kitada M, Ishikawa H, Itokazu Y, Wakao S, Dezawa M. Peripheral nerve regeneration by the in vitro differentiatedhuman bone marrow stromal cells with Schwann cell property. Biochem Biophys Res Commun 2007; 359(4): 915-20.

[78] Wakao S, Hayashi T, Kitada M, et al. Long-term observation of auto-cell transplantation in non-human primate reveals safety and efficiency of bone marrow stromal cell-derived Schwann cells in peripheral nerve regeneration. Exp Neurol 2010; 223(2): 537-47.

[79] Bongso A, Fong CY, Gauthaman K. Taking stem cells to the clinic: Major challenges. J Cell Biochem 2008; 105(6): 1352-60.

[80] Can A, Karahuseyinoglu S. Concise review: human umbilical cord stroma with regard to the source of fetus-derived stem cells. Stem Cells 2007; 25(11): 2886-95.

[81] Wu KH, Zhou B, Lu SH, et al. In vitro and in vivo differentiation of human umbilical cord derived stem cells into endothelial cells. $J$ Cell Biochem 2007; 100(3): 608-16.

[82] Conconi MT, Burra P, Di Liddo R, et al. CD105(+) cells from Wharton's jelly show in vitro and in vivo myogenic differentiative potential. Int J Mol Med 2006; 18(6): 1089-96.

[83] Fu YS, Shih YT, Cheng YC, Min MY. Transformation of human umbilical mesenchymal cells into neurons in vitro. J Biomed Sci 2004; 11(5): 652-60.

[84] Nishiyama $\mathrm{N}$, Miyoshi $\mathrm{S}$, Hida $\mathrm{N}$, et al. The significant cardiomyogenic potential of human umbilical cord blood-derived mesenchymal stem cells in vitro. Stem Cells 2007; 25(8): 2017-24.

[85] Mitchell KE, Weiss ML, Mitchell BM, et al. Matrix cells from Wharton's jelly form neurons and glia. Stem Cells 2003; 21(1): 5060 .

[86] Anzalone R, Lo Iacono M, Loria $\mathrm{T}$, et al. Wharton's jelly mesenchymal stem cells as candidates for beta cells regeneration: extending the differentiative and immunomodulatory benefits of adult mesenchymal stem cells for the treatment of type 1 diabetes. Stem Cell Rev 2011; 7(2): 342-63.

[87] Anzalone R, Lo Iacono M, Corrao S, et al. New emerging potentials for human Wharton's jelly mesenchymal stem cells immunological features and hepatocyte-like differentiative capacity. Stem Cells Dev 2010; 19(4): 423-38.

[88] $\mathrm{Xu} \mathrm{Q}$, Zhang $\mathrm{HT}$, Liu $\mathrm{K}$, et al. In vitro and in vivo magnetic resonance tracking of Sinerem-labeled human umbilical mesenchymal stromal cell-derived Schwann cells. Cell Mol Neurobiol 2011; 31(3): 365-75.

[89] Gimble J, Guilak F. Adipose-derived adult stem cells: isolation, characterization, and differentiation potential. Cytotherapy 2003; 5(5): 362-9.

[90] Gimble JM, Katz AJ, Bunnell BA. Adipose-derived stem cells for regenerative medicine. Circ Res 2007; 100(9): 1249-60.

[91] Puglisi MA, Saulnier N, Piscaglia AC, Tondi P, Agnes S, Gasbarrini A. Adipose tissue-derived mesenchymal stem cells and hepatic differentiation: old concepts and future perspectives. Eur Rev Med Pharmacol Sci 2011; 15(4): 355-64.

[92] Baer PC. Adipose-Derived Stem Cells and Their Potential to Differentiate into the Epithelial Lineage. Stem Cells Dev. 2011 
[93] Radtke C, Schmitz B, Spies M, Kocsis JD, Vogt PM. Peripheral glial cell differentiation from neurospheres derived from adipose mesenchymal stem cells. Int J Dev Neurosci 2009; 27(8): 817-23.

[94] Wei Y, Gong K, Zheng Z, et al. Schwann-like cell differentiation of rat adipose-derived stem cells by indirect co-culture with Schwann cells in vitro. Cell Prolif 2010; 43(6): 606-16.

[95] Hung SC, Cheng H, Pan CY, Tsai MJ, Kao LS, Ma HL. In vitro differentiation of size-sieved stem cells into electrically active neural cells. Stem Cells 2002; 20(6): 522-9.

[96] Neshati Z, Matin MM, Bahrami AR, Moghimi A. Differentiation of mesenchymal stem cells to insulin-producing cells and their impact on type 1 diabetic rats. J Physiol Biochem 2010; 66(2): 181-7.

[97] Johnson JE, Zimmerman K, Saito T, Anderson DJ. Induction and repression of mammalian achaete-scute homologue (MASH) gene expression during neuronal differentiation of P19 embryonal carcinoma cells. Development 1992; 114(1): 75-87.

[98] Takahashi J, Palmer TD, Gage FH. Retinoic acid and neurotrophins collaborate to regulate neurogenesis in adult-derived neural stem cell cultures. J Neurobiol 1999; 38(1): 65-81.

[99] von Holst A, Rodriguez-Tebar A, Michaille JJ, et al. Retinoic acidmediated increase in TrkA expression is sufficient to elicit NGFdependent survival of sympathetic neurons. Mol Cell Neurosci 1995; 6(3): 185-98.

[100] Jessen KR, Mirsky R. The origin and development of glial cells in peripheral nerves. Nat Rev Neurosci 2005; 6(9): 671-82.

[101] Chaudhary LR, Avioli LV. Activation of extracellular signalregulated kinases 1 and 2 (ERK1 and ERK2) by FGF-2 and PDGF$\mathrm{BB}$ in normal human osteoblastic and bone marrow stromal cells: differences in mobility and in-gel renaturation of ERK1 in human, rat, and mouse osteoblastic cells. Biochem Biophys Res Commun 1997; 238(1): 134-9.
[102] Davis JB, Stroobant P. Platelet-derived growth factors and fibroblast growth factors are mitogens for rat Schwann cells. J Cell Biol 1990; 110(4): 1353-60.

[103] Kim HA, Ratner N, Roberts TM, Stiles CD. Schwann cell proliferative responses to cAMP and Nf1 are mediated by cyclin D1. J Neurosci 2001; 21(4): 1110-6.

[104] McKenzie IA, Biernaskie J, Toma JG, Midha R, Miller FD. Skinderived precursors generate myelinating Schwann cells for the injured and dysmyelinated nervous system. J Neurosci 2006; 26(24): 6651-60.

[105] Dezawa M. Systematic neuronal and muscle induction systems in bone marrow stromal cells: the potential for tissue reconstruction in neurodegenerative and muscle degenerative diseases. Med Mol Morphol 2008; 41(1): 14-9.

[106] McGuirk JP, Weiss ML. Promising cellular therapeutics for prevention or management of graft-versus-host disease (a review). Placenta 2011; 32(Suppl 4): 5304-10.

[107] Kamada T, Koda M, Dezawa M, et al. Transplantation of human bone marrow stromal cell-derived Schwann cells reduces cystic cavity and promotes functional recovery after contusion injury of adult rat spinal cord. Neuropathology 2011;31(1): 48-58.

[108] Kamada T, Koda M, Dezawa M, et al. Transplantation of bone marrow stromal cell-derived Schwann cells promotes axonal regeneration and functional recovery after complete transection of adult rat spinal cord. J Neuropathol Exp Neurol 2005; 64(1): 37-45.

[109] Someya Y, Koda M, Dezawa M, et al. Reduction of cystic cavity, promotion of axonal regeneration and sparing, and functional recovery with transplanted bone marrow stromal cell-derived Schwann cells after contusion injury to the adult rat spinal cord. J Neurosurg Spine 2008; 9(6): 600-10.

(C) Kuroda et al.; Licensee Bentham Open.

This is an open access article licensed under the terms of the Creative Commons Attribution Non-Commercial License (http://creativecommons.org/ licenses/by-nc/3.0/), which permits unrestricted, non-commercial use, distribution and reproduction in any medium, provided the work is properly cited. 